\title{
La dimensione ecclesiale del presbitero diocesano
}

The Ecclesial Dimension of the Diocesan Priest

ERIO CASTELLUCCI

Arcivescovo di Modena-Nonantola

doneriocastellucci@gmail.com

Fecha de recepción: 7/12/2021

Fecha de aceptación: 30/12/21

https://doi.org/10.52039/seminarios.v66vi229.890

SOMMARIO: La dimensione ecclesiale è essenziale per il presbitero diocesano, così come quella cristologica, dalla quale dipende. Egli è infatti il cristiano chiamato (vocazione) e abilitato (ordinazione) a dedicarsi completamente all'edificazione della Chiesa, attraverso il ministero dell'annuncio della parola di Dio, della celebrazione dei sacramenti e della guida pastorale. Questo triplice compito implica l'inserimento nella rete di relazioni di una Chiesa locale, e concretamente nella relazione di corresponsabilità con il popolo di Dio profetico, sacerdotale e regale, con il presbiterio e con il vescovo. Povertà, castità e obbedienza, nella loro declinazione ministeriale, se vissute nella carità pastorale, favoriscono una donazione piena e gioiosa.

PAROLE-CHIAVE: Comunione ecclesiale, Chiesa locale, ministero ordinato, carità pastorale.

ABSTRACT: The ecclesial dimension is essential for the diocesan priest, as is the Christological dimension, on which it is rooted. The priest is a Christian called (vocation) and empowered (ordination) to devote himself entirely to the edification of the Church through the ministry of proclaiming the word of God, celebrating the sacraments, and providing pastoral guidance. This triple task implies insertion in the network of the local Church and concretely in a relationship of co-responsibility with the prophetic, priestly and royal people of God, with the presbyterate and with the bishop. Poverty, chastity, and obedience, in their ministerial expression, if lived in pastoral charity, favor a full and joyful donation.

KEYwords: Church Communion, Local Church, Ordained Ministry, Pastoral Charity.

Da più di cinquant'anni, con alterne vicende, la teologia si interroga sull'identità presbiterale. Spesso l'approccio è «essenzialista», nel tentativo - giusto e opportuno - di trovare un «fil rouge» che, dall'epoca apostolica ad oggi, permetta di individuare ciò che permane dentro ai grandi e continui 
cambiamenti. E di solito questa continuità è individuata nella relazione con Cristo (e in lui con la Trinità): è lui, infatti, colui che era, che è e che viene, è lui l'alpha e l'omega (Ap 22,13), colui che rimane in eterno. Sempre di più, tuttavia, emerge nella teologia e nella vita pastorale la convinzione che l'identità presbiterale comporta indissolubilmente anche la relazione alla comunità, ossia la dimensione ecclesiale; e non semplicemente la relazione con la Chiesa in generale, ma in essa con la Chiesa particolare, la diocesi. In fondo la riflessione del Vaticano II, sia nella Costituzione sulla Chiesa Lumen Gentium (n. 28), sia nel decreto Presbyetrorum Ordinis, ha cercato proprio di integrare l'aspetto ecclesiologico del ministero, che era passato in sordina nel secondo millennio, con quello cristologico, che invece era stato così esaltato da dare origine alla spiritualità del sacerdos alter Christus: espressione, non a caso, evitata dal Concilio. ${ }^{1}$

La riflessione che segue tenta di mettere in luce, all'interno dell'identità presbiterale, la connessione tra le due dimensioni: il rapporto con Cristo e l'inserimento vitale nella Chiesa particolare. Non saranno concetti nuovi per chi pratica la teologia del ministero; speriamo però che siano utili almeno a recuperare l'integrazione tra due aspetti che troppe volte vengono considerati separatamente, dando perfino origine a contrapposizioni tra chi, da una parte, esalta talmente la relazione con Cristo da ricondurre il ministero ad una «mediazione tra il cielo e la terra» e chi, dal versante opposto, elimina ogni riferimento cristologico e propone una semplice leadership ecclesiastica.

\section{SGUARDo TEOLOGICO-PASTORALE SUI PRESBITERI ALL'INIZIO DEL TERZO MILLENNIO}

L'identità teologica del presbiterato è un dato costante nei suoi elementi sostanziali, ma necessariamente mutevole e mai compiutamente raggiunto nelle sue configurazioni storiche e versioni operative. Da una parte infatti, in quanto «secondo grado» del sacramento dell'ordine, si radica nel ministero apostolico affidato da Cristo agli apostoli e trasmesso da questi ai loro successori, i vescovi, i quali a loro volta ne hanno reso partecipi come loro collaboratori i presbiteri; il ministero presbiterale, in comunione con quello episcopale, rappresenta efficacemente «nella» Chiesa e «per la» Chiesa la presenza e azione di Cristo capo, sposo e pastore, attraverso la predicazione

1. La bibliografia è amplissima; mi limito a indicare due contributi importanti, usciti subito dopo la conclusione del Vaticano II. Il primo, brevissimo, di Y. Congar, «L'idée du sacerdoce dans les textes de Vatican II», Verbum Caro 85 (1968) 11-15; più ampio quello di J. Frisque, «Théologie du sacerdoce selon Vatican II», Revue Nouvelle (1966) 481-496. 
della parola di Dio, la celebrazione dei sacramenti e la guida pastorale del popolo di Dio. Dall'altra parte - e fatti salvi questi elementi - il presbiterato si plasma sempre nuovamente, e con accentuazioni diverse, sulla concreta configurazione sociale ed ecclesiale nella quale vive ed opera, con le sue luci ed ombre, opportunità e resistenze: di qui la necessità di «aggiornare» periodicamente la teologia e la spiritualità dei presbiteri, e di qui anche la legittima varietà delle figure presbiterali che popolano la storia e la geografia della Chiesa in tutto il mondo.

Se negli ultimi cinque secoli, dal Concilio di Trento in avanti, è il «modello pastorale» che ha connotato teologia, ministero e spiritualità dei presbiteri, restano numerose sia le versioni concrete da esso assunte sia le possibilità di vivere ed esercitare il presbiterato seguendo altre sottolineature. ${ }^{2}$ Per questo convivono legittimamente e si completano a vicenda, nelle Chiese del mondo, presbiteri che interpretano il loro ministero in chiave prevalentemente liturgica e sacramentale, con altri che mettono al primo posto la testimonianza della carità nelle situazioni disagiate; presbiteri che si dedicano essenzialmente all'animazione della comunità cristiana e alla pastorale cosiddetta ordinaria, con altri che accolgono le sfide dei «nuovi areopaghi» della cultura e comunicazione, percorrendo le diverse possibilità offerte oggi dai mass media in tutti i campi; presbiteri che puntano soprattutto sulle relazioni interpersonali e si pongono come accompagnatori e guide spirituali, con altri che spendono le loro migliori energie nella formazione o nell'insegnamento della religione, teologia e scienze umane o magari, almeno per certi periodi e in certe situazioni, assumono compiti di supplenza nei campi dell'educazione, promozione umana e professionale o altro.

La coniugazione fra elementi sostanziali immutabili e declinazioni storiche mutevoli non è sempre facile: a volte infatti, nel corso della storia anche recente della Chiesa, il presbiterato è stato compreso e vissuto in maniera troppo arroccata sul passato o, viceversa, troppo proiettata verso incerte sperimentazioni. La cosiddetta «crisi di identità» dei presbiteri, che esplose pochi anni dopo la conclusione del Vaticano II, dall'inizio degli anni '70 del secolo scorso, e fu ritenuta sostanzialmente conclusa un ventennio dopo da Giovanni Paolo II, si può interpretare oggi anche come un grande travaglio alla ricerca di una adeguata articolazione fra dogma e storia nella definizione teologica del presbiterato. ${ }^{3}$

2. H. Denis, «La teologia del presbiterato da Trento al Vaticano II», in Y. Congar - J. Frisque (eds.), I preti. Formazione, ministero e vita, Roma 1970, 105-154.

3. Per una panoramica sulle discussioni seguite al Vaticano II e sulla 'crisi di identità', rimando al mio manuale Il ministero ordinato, Brescia 2006, 248-270. 


\section{UNA NUOVA «CRISI DI IDENTITÀ»?}

Per comprendere l'inserimento dei presbiteri nella vita della Chiesa, è opportuno lasciarsi interrogare da alcuni disagi che oggi tra i presbiteri emergono più chiaramente rispetto ad alcuni decenni fa: si tratta di situazioni ed episodi che, sebbene con modalità e intensità non paragonabili alla crisi di identità esplosa mezzo secolo fa, continuano ad interessare le nostre Chiese. Sarebbe esagerato oggi parlare di nuova crisi di identità dei presbiteri: e tuttavia da più parti si segnalano appesantimenti e fatiche, fino alla disaffezione e disillusione, sia in rapporto alla sensibile diminuzione numerica del clero, in molte parti del mondo, a fronte delle enormi esigenze, quantitative e qualitative, espresse dal popolo di Dio, sia in relazione alle non isolate situazioni di disagio che si manifestano specialmente in giovani presbiteri e che sfociano in alcuni casi nella laicizzazione e nell'abbandono del ministero sacerdotale e in altri nella sacralizzazione e nella perdita dell'orizzonte missionario, con una sorta di rifugio nelle pratiche cultuali o di ricerca del piccolo gruppo gratificante.

Da una parte, infatti, i presbiteri già inseriti da un certo tempo nel ministero risentono evidentemente dell'accrescimento dell'età media e del correlato svigorimento di energie: vedono i tanti e grandi bisogni della gente ma non riescono a farvi fronte come vorrebbero; percepiscono la necessità di suscitare e formare collaboratori laici ma non trovano sufficiente tempo, motivazioni e forze per dedicarvisi, anche a motivo dei molteplici adempimenti di carattere amministrativo e giuridico che continuano a pesare su di loro.

Dall'altra parte, le crisi denunciate da alcuni presbiteri talvolta pochissimo tempo dopo l'ordinazione, sembrano risultare da diverse cause: la fragilità psicologica e affettiva da cui sono segnati in generale giovani di oggi, e quindi anche quelli che intraprendono la strada del Seminario; il clima culturale odierno, che appare legato ad un'idea di libertà istintiva e quindi piuttosto refrattario nei confronti di ogni decisione che si presenti come definitiva, privilegiando al contrario le scelte -anche generose- dalle quali si possa retrocedere quando si presentino difficoltà; le stesse situazioni pastorali sempre più complesse, che mettono alla prova il carattere, la maturità, la personalità e la formazione teologica del presbitero; infine, la formazione seminaristica e permanente, che può apparire talora sganciata dalla realtà e tesa a delineare un «ideale» di presbitero compatto e monolitico, che non trova poi riscontri nella concreta attività pastorale, nella quale occorre articolare sapientemente i contenuti teorici tenendo conto della estrema varietà delle situazioni. 
È di fronte a queste sfide, oggi particolarmente evidenti, che la Chiesa, in piena fedeltà al Magistero universale, è chiamata a confrontare i dati rivelati che appartengono alla struttura perenne del presbiterato con le situazioni sempre nuove che contribuiscono a plasmarne la figura. Le singole Chiese locali poi, con le loro differenti realtà sociali e pastorali, costituiscono l'ultima mediazione e, per così dire, il filtro decisivo attraverso il quale prende forma concreta il ministero presbiterale in questo luogo e in questo tempo.

\section{IL MINISTERO PRESBITERALE NELLA CHIESA MISTERO, COMUNIONE E MISSIONE}

Il presbiterato è un ministero ecclesiale e i presbiteri sono quindi, prima di tutto, dei credenti in Cristo che appartengono vitalmente alla sua comunità, la Chiesa: la domanda sull'identità dei presbiteri si colloca dunque entro la domanda, più ampia, sull'identità della Chiesa. Come già sopra accennato, è stato il Concilio Vaticano II che, dopo secoli di prevalenza di un modello sacerdotale quasi esclusivamente «cristologico», ha reinserito il ministero presbiterale in una dimensione anche «ecclesiologica». Ogni vocazione, carisma e ministero, nella visione conciliare, si radica nel battesimo e nell'eucaristia e rappresenta una particolare modalità di incarnare e svolgere la missione ecclesiale. È quindi opportuno richiamare per cenni alcuni essenziali aspetti della natura della Chiesa, dalla cui considerazione emergerà poi la specifica modalità della missione presbiterale al suo interno.

Per entrare nella vita intima della Chiesa, le coordinate più adatte non sono quelle desunte dalla sociologia, ma quelle tipicamente teologiche di mistero, comunione e missione. ${ }^{4}$ La Chiesa è convocata, compaginata e inviata dalla Trinità. Ogni attività ecclesiale si muove dentro a questa vita intima di mistero, comunione e missione: l'annuncio della parola di Dio, la celebrazione dei sacramenti e la vita di carità la esprimono e rafforzano. La Chiesa annuncia ciò che essa stessa ascolta, come serva fedele della parola che le è donata: e se il suo annuncio orienta gli uomini a rispondere alla chiamata di Dio, ad entrare nella comunione ecclesiale e a diventare a loro volta testimoni per altri, è perché essa per prima si è lasciata convocare, formare e inviare dalla parola che annuncia. La Chiesa celebra ciò da cui essa stessa è compaginata: e se la celebrazione dei sacramenti - specialmente dell'eucaristia - offre agli uomini la grazia santificante che li chiama alla

4. S. Dianich, Teologia del ministero ordinato, Paoline, Roma 1984. 
salvezza, li rende popolo di Dio, corpo di Cristo e tempio dello Spirito, e li spinge alla missione, è perché essa per prima si è lasciata radunare, edificare e vivificare dai sacramenti che celebra. La Chiesa, infine, dona e testimonia la carità da cui essa stessa è plasmata: e se la sua molteplice opera caritativa, in campo educativo, culturale, sociale, politico, si pone tra gli uomini come segno efficace dell'amore e della giustizia di Dio che chiama e raduna alla salvezza già a partire da questa vita e invia gli uomini a proclamarla a tutti, è perché essa per prima si è lasciata chiamare, radunare e inviare dallo Spirito, amore sostanziale del Padre e del Figlio, di cui essa stessa è frutto.

L'appartenenza viva alla Chiesa è dunque dono prima che compito. La perdita della consapevolezza che essere Chiesa è prima di tutto dono, frutto di una chiamata - ciò che è scritto nell'etimologia stessa di Chiesa-ekklesia, convocazione - porterebbe a visioni ecclesiologiche e pastorali distorte. La coscienza di essere con-vocazione infatti è per la Chiesa condizione essenziale della sua esistenza e attività: essa sa di non essere autogena, ma di ricevere continuamente i doni dai quali è costituita e che offre agli uomini: Parola, sacramenti e carità: ella sa che questi doni altro non sono se non la presenza attiva del Risorto, di colui che è via, verità e vita (Gv 14,6), di colui che è l'Inviato del Padre che invia lo Spirito.

Conseguentemente le immagini che nel Nuovo Testamento si applicano alla Chiesa sono per la maggior parte di carattere cristologico e mettono in evidenza il legame della Chiesa con Gesù: tali sono - per richiamare le principali dagli scritti giovannei e paolini - quelle del gregge e del pastore (Gv 10,1-19), dei tralci e della vite ( Gv 15,1-11), della sposa e dello sposo (Ef 5,21-33), del corpo e del capo (Ef 1,22-23; 4,15-16; 5,23 e Col 1,18). Queste immagini evidenziano, rispettivamente, che la Chiesa è protetta e curata da Cristo e senza di lui soccomberebbe, è inserita nella vita stessa di Gesù, senza la quale inaridirebbe, è originata e resa feconda dall'amore di Cristo, senza il quale sarebbe sterile, è guidata e illuminata dal Figlio di Dio, senza il quale si ridurrebbe ad un corpo inerte. Sono quindi immagini che esprimono la consapevolezza del carattere preveniente della grazia di Cristo rispetto alla Chiesa: è lui che la tiene in vita, dando se stesso per lei (Gv 10,11. 15. 17-18; Ef 5,25), trasmettendole continuamente la linfa (Gv 15,4-5) e costituendone il centro vitale e operativo (Ef 4,15-16). La natura della Chiesa è di farsi grembo accogliente della grazia di Cristo: grembo aperto a tutti gli uomini. È quanto esprime, tra l'altro, il motivo biblico-patristico, ripreso dal Vaticano II, di Maria come icona e specchio della Chiesa. 


\section{SACERdozio battesimale E SACERDOZIO Ministeriale NELla CHIESA}

L'insistenza sul carattere accogliente della Chiesa rispetto alla grazia di Cristo non deve far dimenticare la necessità di una risposta da parte della Chiesa a questa stessa grazia: ciascun battezzato, e la Chiesa nel suo insieme, sono invitati pressantemente, da ogni pagina della Scrittura e della Tradizione, ad una risposta generosa all'offerta di grazia che Dio, in Cristo e nello Spirito, presenta attraverso la sua parola, i sacramenti e i doni dello Spirito. La risposta ecclesiale, che comprende le tre virtù teologali della fede, speranza e carità (1 Ts 1,2-3; 5,8-10; 1 Cor 13,13; Col 1,4-5), si può esprimere attraverso la categoria del sacerdozio battesimale. Paolo chiama «culto spirituale» l'atteggiamento dei battezzati a offrire i loro «corpi come sacrificio vivente, santo e gradito a Dio» (Rom 12,1): quale risposta migliore, all'offerta di sé da parte del Figlio, dell'offerta di noi stessi -nella concretezza delle nostre relazioni-a Dio? I cristiani, così, ereditano l'antica promessa di diventare un regno di sacerdoti (Es 19,6 con Ap 1,6 e 5,10; 20,6) e, in virtù del battesimo, sono essi stessi «sacerdozio santo e regale» $(1 \mathrm{Pt} 2,5.9)$. Siamo giunti al nucleo della Chiesa anche dal punto di vista della risposta: il dono di sé, l'offerta della propria vita come sacrificio a Dio, che si compie e rinnova nella celebrazione dell'eucaristia, dove il sacrificio dei cristiani viene assunto nel perfetto sacrificio di Cristo e innalzato al Padre. I battezzati hanno come scopo - comprensivo di tutti gli altri - quello di offrire se stessi nelle svariate circostanze della vita: il compito sacerdotale di annunciare le grandi opere di Dio, celebrare i sacramenti e vivere la carità è comune a tutti ed è la via della salvezza. ${ }^{5}$

La fantasia dello Spirito, però, dispone che ciascuno viva e realizzi il sacerdozio comune secondo modalità differenti, suggerite dalle svariate vocazioni, dai vari carismi, dai diversi servizi e ministeri (1 Cor 12,8-10; 28-30; 14,26; Rom 12,6-8; Ef 4,11). La terminologia neotestamentaria è ancora fluttuante, mancando l'interesse per una vera e propria strutturazione dei ministeri: sia perché durante la loro vita gli apostoli stessi si consideravano ed erano ritenuti i ministri delle comunità da loro fondate, sia per un clima diffuso di attesa del ritorno imminente di Cristo. ${ }^{6}$ Già nelle Lettere Pastorali, tuttavia, si registra una accentuazione istituzionale; e ben presto, a partire dai Padri apostolici e soprattutto dalle Lettere di Ignazio di Antiochia, l'attenzione si concentra su

5. P. Grelot, «Il sacerdozio comune dei fedeli nel Nuovo Testamento», in Idem, Regole e tradizioni del cristianesimo primitivo, Casale Monferrato 1998, 115-131.

6. R. Penna, «Los ministerios en las primeras comunidades cristianas según el Nuevo Testamento», Seminarios 66 (2021) 11-33, pp. 17-28. 
tre di essi - episcopato, presbiterato e diaconato - che diventano poi a poco a poco i «gradi» del ministero ordinato. La tradizione ecclesiale, quindi, ravviserà in questi tre ministeri il compito di garantire il radicamento apostolico della Chiesa. Il gesto dell'imposizione delle mani, che già nel Nuovo Testamento viene utilizzato anche come trasmissione del potere ministeriale (At 6,6; 1 Tim 4,14; 5,22; 2 Tim 1.6), diventerà poi nella tradizione ecclesiale il segno efficace-sacramentale dell'inserimento di un uomo nella «tradizione apostolica». I ministeri ordinati costituiscono in sintesi, nella vita della Chiesa, il segno e la garanzia della sua apostolicità, assicurando alla comunità il legame con le sue origini apostoliche: essi si basano non semplicemente sulle qualità umane di chi viene eletto, ma sul conferimento da parte del Signore, attraverso la Chiesa, di un dono che li pone anche «di fronte» alla comunità, come segno efficace della grazia.

\section{IL MiNISTERO NELLA CHIESA UNIVERSALE E PARTICOLARE}

Il ministero ordinato, che ha il suo apice nell'episcopato e che dai vescovi è stato trasmesso in vari gradi ai loro collaboratori, porta in sé quindi una dimensione dell'apostolicità che non è assorbita dall'intera Chiesa: gli apostoli furono chiamati, raccolti e inviati da Cristo non solo come «il seme del nuovo Israele», ma anche come «l'origine della sacra gerarchia» (Ad Gentes 5); il multiforme potere -da esercitare come diakonia nel nome di Gesù che è venuto per servire e non per essere servito (Mc 10,42-45 par.)- di annunciare, battezzare, perdonare i peccati, ripetere il gesto eucaristico, edificare e guidare la comunità, ricevuto da Cristo, implicava anche un esercizio dell'apostolato per la comunità; e come tale venne inteso sia nella pratica apostolica sia nella riflessione sull'apostolato (in particolare: At 1,17.25; 2 Cor 3,6. 8-9; 2 Cor 5,18; 6,4; Col 1,25; Ef 3,7).

Agli Apostoli Gesù dà quindi anche un compito ministeriale, che consiste nel perpetuarne la diakonia, attualizzando la sua presenza nella Chiesa e nel mondo. «Tutti i giorni, fino alla fine del mondo» (Mt 28,20) Cristo è presente come crocifisso risorto, e come tale raccoglie egli stesso, nello Spirito, la sua Chiesa anche oggi: ma proprio perché la Chiesa mantenga la coscienza di essere raccolta dal Signore e da lui continuamente dotata dei doni di cui ha bisogno per vivere e operare -coscienza, come dicemmo, essenziale perché la Chiesa sia davvero tale-Cristo ha stabilito in essa un segno efficace della priorità della sua grazia rispetto ad ogni risposta umana. Il ministero ordinato è perciò quel carisma che, radicato sul servizio apostolico, ricorda alla Chiesa che la grazia di Cristo la previene, e che opera in modo da far sperimentare 
alla comunità la grazia come «dono» prima che come «compito». In questo senso esso è continuazione nella storia dell'unica missione salvifica di Cristo a favore degli uomini. Il ministro ordinato, in altre parole, è il cristiano che si dedica stabilmente e totalmente, in nome di Cristo pastore, capo e sposo, all'edificazione della Chiesa.

Ciò implica che la dimensione locale-particolare della Chiesa entra nell'essenza stessa del ministero ordinato: la parola di Dio, infatti, risuona in una comunità concreta e qui opera adesioni, conversioni e santità; i sacramenti sono nello stesso tempo opera di Cristo e della Chiesa, e specialmente la celebrazione eucaristica sta all'origine, al centro e al culmine della vita delle comunità; i doni dello Spirito, e soprattutto la carità, esigono per essere riconosciuti ed esercitati una trama di relazioni visibili e incarnate. In ciascuna Chiesa particolare, raccolta attorno al proprio vescovo in comunione con il vescovo di Roma, è presente lo stesso Cristo nello Spirito, ma in modi sempre diversi, perché diverse sono le situazioni delle Chiese nelle quali risuona la Parola, vengono celebrati i sacramenti e vissuti i carismi. Nessuna Chiesa particolare è semplicemente la copia di un'altra. Ogni Chiesa particolare apporta alla Cattolica il contributo del proprio genius loci, di culture, tradizioni, figure di santità, situazioni sociali, problemi e risorse: si può parlare in proposito di una spiritualità diocesana, nella quale e servizio della quale si collocano i ministri ordinati incardinati in una Chiesa particolare o in essa operanti. Il ministero ordinato è dunque non solo «nella» Chiesa ma «in quella» Chiesa, di cui respira la spiritualità e verso la quale esprime la diakonia, come segno efficace di Cristo pastore, capo e sposo.

\section{LA CARITÀ PASTORALE, CHIAVE Di LETTURA DEL Ministero E DELLA VITA DEI PRESBITERI}

Il ministero ordinato è in tal modo caratterizzato dalla carità pastorale, come nota teologica; stile operativo e via propria di santità. ${ }^{7}$ Ogni battezzato, in forza dei sacramenti, è chiamato a santificarsi nella sequela ed imitazione di Cristo; la diversità delle vocazioni, situazioni ed esperienze, dei carismi e ministeri, fa sì che l'unica vocazione cristiana, quella alla santità attraverso la carità, venga vissuta in maniera differente da ciascun cristiano. Si individuano tuttavia, tra la chiamata alla santità comune a tutti i battez-

7. Attorno alla nozione di 'carità pastorale' ruota l'Esortazione post-sinodale Pastores dabo vobis (1992) di papa Giovanni Paolo II; C. Dumont, «La 'charite pastorale' et la vocation au presbytérat. A propos de l'Exhortation post-synodale Pastores dabo vobis», Nouvelle Revue Théologique 115 (1993) 211-226. 
zati e le differenti traduzioni nella vita di ciascuno, alcune piste vocazionali fondamentali: la chiamata alla vita laicale, che ha una consistenza già in se stessa in quanto testimonianza della presenza del regno di Dio nelle realtà temporali, e che spesso sfocia nella chiamata al matrimonio e alla famiglia e talvolta nella consacrazione secolare; la chiamata al sacerdozio ministeriale, che testimonia efficacemente nella Chiesa la priorità della grazia di Cristo pastore, capo e sposo, dalla quale la Chiesa stessa è edificata; la chiamata alla consacrazione religiosa, che testimonia la trascendenza del regno di Dio rispetto a qualunque valore o realizzazione degli uomini e inserisce nella storia e nella società il dono del richiamo all'eternità. Queste piste determinano altrettante forme concrete della carità, che si implicano e si arricchiscono a vicenda: esistono quindi forme di carità che potremmo definire laicale, matrimoniale-familiare, monastica, religiosa... e pastorale. La carità pastorale, così intesa, è la concreta forma di amore attraverso cui si santifica il battezzato al quale è trasmesso il sacerdozio ministeriale.

La carità pastorale rimanda, in primo luogo, a Cristo pastore, origine, modello e linfa del ministero presbiterale. In questo senso, la carità pastorale mette in luce come la sorgente del presbiterato si collochi non nelle qualità umane, morali, intellettuali, spirituali di un uomo, e neppure semplicemente in un riconoscimento ecclesiale, bensì in una chiamata ed abilitazione che vengono da Cristo. E' da questa prospettiva che il carattere va riaffermato come configurazione a Cristo sacerdote in modo da poter agire in persona di Cristo capo. La forza per svolgere efficacemente il ministero presbiterale proviene dalla fedeltà di Dio, sigillata dal dono spirituale che, attraverso il sacramento dell'ordine, riposa sul presbitero in maniera permanente. $\mathrm{Si}$ tratterà quindi solamente di «ravvivare il dono» che è stato trasmesso per l'imposizione delle mani (2 Tim 2,6).

In quanto segni viventi di Cristo pastore, i presbiteri sono chiamati a riproporne l'atteggiamento oblativo «per» la Chiesa e abilitati ad essere strumenti efficaci per la sua edificazione, attraverso la parola, i sacramenti e la carità. Si potrebbe riflettere anche sul legame tra il presbitero e Cristo capo o Cristo sposo, ma le conclusioni andrebbero nella stessa direzione: nella comunità cristiana, sotto la guida del vescovo, i presbiteri sono testimoni efficaci della grazia preveniente con la quale Cristo risorto edifica continuamente la Chiesa.

In secondo luogo, la carità pastorale rimanda alle relazioni oggettive che il presbitero vive «nella» Chiesa. Il ministero e la vita spirituale del presbitero trovano in Cristo pastore, capo e sposo la loro fonte originaria e permanente, e nella trama dei rapporti ecclesiali il luogo concreto della loro crescita. La 
dimensione ecclesiale del presbiterato, come si è visto, è già implicitamente compresa nel fatto che il presbitero è configurato a Gesù Cristo in quanto capo e pastore della Chiesa; di Cristo, cioè, che si relaziona alla Chiesa: sarebbero in qualche modo incompleti un pastore senza gregge e un capo senza corpo, come anche uno sposo senza sposa. Il presbiterato è quindi per il ministero ecclesiale e non per una dignità personale. La valenza pastorale della carità presbiterale, allora, non si esaurisce nel rapporto sacramentale, intimo e vivo con Cristo pastore - rapporto che rimane sempre alla base - ma si innerva anche ed essenzialmente nelle relazioni ecclesiali; l'aggettivo «pastorale» va dunque inteso non solo nei termini soggettivi di una disponibilità personale generosa, ma anche nei termini oggettivi di un intreccio di relazioni all'interno del popolo di Dio, di un popolo che abita la Chiesa particolare.

\section{CARità PAStorale E Chiesa PARTicolare}

I presbiteri sono chiamati a vivere una relazione filiale con il proprio vescovo (e, attraverso di lui, con il papa) del quale sono provvidi collaboratori e che è loro padre e guida; una relazione fraterna con gli altri presbiteri e in particolare con quelli della medesima Chiesa particolare, insieme ai quali formano un unico presbiterio, il quale come «corona del vescovo» partecipa dell'unico ministero e richiede stima reciproca, comunione e collaborazione, che possono esprimersi anche in svariate forme di vita comune ; una relazione paterna e materna con i laici, ai quale sono inviati, siano essi appartenenti o meno alla Chiesa, membra vive, collaboratori, diaconi e ministri istituiti, oppure battezzati che non prendono parte alla vita comunitaria.

La coltivazione di questa triplice relazione, nei presbiteri, passa attraverso la sintonia con le scelte formative, operative e missionarie che una Chiesa particolare compie e porta avanti: se è davvero «pastorale», la carità del presbitero non è mai privata, avulsa dal concreto cammino missionario della sua Chiesa; atteggiamenti anche generosi di dedizione possono essere depotenziati o vanificati da individualismi che finiscono per dividere. Ciò non significa livellare i diversi stili che può assumere il ministero dei presbiteri: significa piuttosto mettere la diversità al servizio dell'edificazione comune.

È dunque l'intera Chiesa particolare che, nello stile sinodale del discernimento comunitario, sotto la guida del vescovo in comunione con il papa, individua nella propria situazione le priorità pastorali secondo le quali modellare concretamente la propria comunione e missione: il presbitero offrirà il dono dell'annuncio, celebrazione e guida inserendosi creativamente e fedelmente nelle scelte della Chiesa locale nella quale è incardinato od opera. 
La figura del presbitero diocesano evidenzia in modo paradigmatico come l'appartenenza e dedicazione ad una Chiesa particolare sia un vero e proprio «valore» spirituale. Sono molte le forme antiche e recenti di spiritualità: pensiamo solo alle grandi tradizioni degli ordini monastici, di quelli mendicanti e delle congregazioni moderne e contemporanee, oltre che alla nascente spiritualità familiare e a quelle nate da esperienze di gruppi, associazioni e movimenti. Ebbene, tra queste esiste anche una spiritualità dei presbiteri diocesani: essere incardinati in una Chiesa particolare e dedicarsi stabilmente alla sua edificazione attraverso la triplice diakonia di annuncio, celebrazione e guida, è una vera e propria via di santificazione, un cammino significativo e realizzante per il cristiano. Le altre spiritualità eventualmente abbracciate dal presbitero diocesano potranno arricchire quella diocesana, non rimpiazzarla: essa infatti - nella sua triplice oggettiva relazione con il vescovo, il presbiterio e i laici a cui si è inviati - rimane sempre il perno attorno al quale ruotano le altre. I presbiteri appartenenti a congregazioni e ordini religiosi, poi, sebbene non siano incardinati in una Chiesa particolare, in essa vivono ed operano: e se quella Chiesa riceve da essi il prezioso servizio della testimonianza e del ministero, essi saranno attenti ad inserire il loro carisma nel cammino di comunione e missione che essa compie, in modo che diventi stimolo e ricchezza per tutti.

\section{CARITÀ PASTORALE E UNITÀ NEL MINISTERO ECCLESIALE}

Il ventaglio degli impegni è così ampio e vario che esiste realmente il rischio per il presbitero della dispersione; l'annuncio della parola di Dio può assumere forme molteplici, come la pre-evangelizzazione, l'evangelizzazione e la catechesi, l'omelia e l'insegnamento, l'intervento nei dibattiti e la lectio divina; l'attività liturgica vede i presbiteri impegnati spesso in prima persona nella preparazione e celebrazione dei sacramenti e nella vita di preghiera della comunità cristiana; essi esercitano, infine, la loro guida pastorale anche nel discernimento dei doni e della vocazione di ciascun battezzato, nell'accompagnamento spirituale delle persone loro affidate, nel coordinamento della vita comunitaria, nell'impegno diretto e indiretto verso $\mathrm{i}$ bisognosi: ed essi rimangono comunque i responsabili ultimi anche di molti aspetti organizzativi, amministrativi e burocratici.

Annuncio della parola, celebrazione dei sacramenti e ministero della carità, come servizio all'unico dono triforme di Cristo risorto, sono interconnessi e si richiamano a vicenda, tanto che l'uno non è completo senza l'altro. L'equilibrio fra questi differenti aspetti del ministero si realizza proprio vivendo 
la carità pastorale, sia nella sua dimensione soggettiva di dedizione e generosità - che rende ogni servizio espressione della cura del buon pastore per il suo gregge - sia nella dimensione oggettiva di inserimento nel cammino della propria Chiesa particolare, in comunione con il vescovo, il presbiterio e i laici. È la congiunzione di queste due istanze della carità pastorale, e non la sola personalità psicologica del presbitero, a determinare la forma concreta che il suo ministero assume: vi sono infatti situazioni e momenti che richiedono maggiori energie nel campo dell'annuncio, altri che portano a privilegiare la celebrazione ed altri ancora, infine, che esigono un impegno più grande nei settori dell'accompagnamento, del coordinamento e dell'intervento caritativo. Se la nota di fondo è la carità pastorale, soggettivamente e oggettivamente intesa, i differenti servizi che il presbitero svolge diventano note di un'unica grande sinfonia.

Poiché l'eucaristia è fonte e culmine della vita ed attività della Chiesa, luogo di concentrazione della Parola e di impulso della carità e della missione, la presidenza della celebrazione eucaristica è analogamente, per il presbitero, fonte e culmine dell'intero suo ministero, occasione nella quale egli esercita nella maniera più intensa possibile il compito di rappresentanza di Cristo Sacerdote, agendo in persona di Cristo capo e pastore. La celebrazione eucaristica è quindi per il presbitero alimento continuo della carità pastorale.

\section{InSERIMENTO NELla CHIESA PARTICOLARE E MiSSIONE UNIVERSALE}

Dove si colloca in questo contesto la missione universale, alla quale i presbiteri sono consacrati con l'ordinazione? Se consideriamo il fatto che la celebrazione eucaristica presieduta dal vescovo insieme al presbiterio e al popolo di Dio, rappresenta principale manifestazione della Chiesa, comprendiamo come la missionarietà universale sia connessa ad una Chiesa particolare. Ogni ministero presbiterale è missionario, poiché Chiesa e mondo, fede e incredulità, santità e peccato, trovano il loro confine più intimo e imperscrutabile nel cuore dell'uomo, specialmente oggi, che la facilità delle comunicazioni ha reso il mondo «villaggio globale», così che ogni persona spesso è attraversata da idee, visioni di vita, tonalità religiose molto eterogenee e talvolta incompatibili tra di loro. Ogni uomo ha degli elementi di «Chiesa» e degli elementi di «mondo»: il presbitero incontra quindi sempre, nel suo ministero, Chiesa e mondo intrecciati tra loro.

Il ministero dell'annuncio ad gentes resta comunque validissimo e quanto mai urgente, costituendo in un certo senso il paradigma della missione della Chiesa nel mondo. Per questo i presbiteri che si dedicano interamente 
a tale missione sono preziosi e insostituibili. Anche per essi vale comunque il legame intrinseco con una Chiesa particolare: siano diocesani o religiosi, essi operano per far sorgere o rafforzare una concreta realtà di Chiesa con un volto, una tradizione, una storia, una serie di situazioni e problemi, che richiedono prima di tutto rispetto ed attenzione; i presbiteri diocesani inviati ad gentes, poi, quando rimangono incardinati nella loro Chiesa particolare, esercitano il loro ministero non a nome proprio ma sempre come espressione dell'attività pastorale della Chiesa che li ha inviati e alla quale appartengono, così che è l'intera Chiesa particolare a farsi carico, attraverso quei presbiteri, della missione ad gentes presso una Chiesa sorella già costituita o in via di costituzione. La missione, così esercitata, farà crescere anche la Chiesa che invia e non solo quella che riceve, poiché la fede si rafforza nell'atto stesso di comunicarla. Occorre quindi riscontrare nei candidati al ministero presbiterale sia la volontà di servire la propria Chiesa particolare, sia la disponibilità a esser mandati a esercitare il proprio ministero ovunque sia richiesto, anche oltre la propria diocesi.

\section{CARitÀ PASTORALE, ELEMENTO UNiFiCATORE TRA MINISTERO E VITA SPIRITUALE}

La carità pastorale, ancora, unifica il ministero e la vita spirituale del presbitero. Il ricentramento della santità cristiana sulla carità ha permesso, all'ultimo concilio, rileggere in termini più sereni anche la relazione tra apostolato e vita spirituale dei presbiteri. Come in ogni battezzato la carità unifica azione e contemplazione, così nel presbitero la carità pastorale unifica ministero e vita. La dedicazione stabile ad una Chiesa particolare e, in essa, alla Chiesa universale, è quindi non solo espressione ma anche alimento della vita spirituale dei presbiteri, e l'attività pastorale non solo esprime ma anche alimenta la carità e la fa crescere. La spiritualità presbiterale coniuga dunque i due poli della spiritualità cristiana - della quale è una versione peculiare - nella forma della spiritualità pastorale, cioè nell'azione dello Spirito dentro ad una storia che è fatta di dedicazione al popolo di Dio nelle diverse espressioni che il ministero presbiterale assume.

Si crea conseguentemente una sorta di «circolo virtuoso» fra la Parola assimilata e donata, i sacramenti ricevuti e celebrati, la carità accolta ed esercitata. La santità dei presbiteri cresce sia nell' ascolto che nell'annuncio della parola di Dio: l'ascolto della Parola, infatti, è condizione per poterla annunciare autorevolmente, poiché il presbitero deve essere il primo credente e, nello stesso tempo, il ministero dell'annuncio favorisce nel presbitero stesso 
l'assimilazione di ciò che proclama agli altri. La santità dei presbiteri cresce poi sia nella recezione che nella celebrazione e presidenza dei sacramenti: accogliere i sacramenti, infatti, è condizione per poterli celebrare vitalmente e, nello stesso tempo, il ministero dei sacramenti favorisce nel presbitero stesso la conformazione a ciò che celebra. La santità dei presbiteri, infine, cresce sia nell'accoglienza che nell'esercizio della carità: l'accoglienza del «frutto dello Spirito» (Gal 5,22), infatti, è condizione per l'inserimento umile e autorevole nella comunità cristiana e, nello stesso tempo, il ministero della guida e del discernimento favorisce nel presbitero stesso l'accoglienza di ciò che testimonia agli altri.

La cura per la propria spiritualità comporta quindi, per il presbitero, la ricerca di un'armonia proporzionata tra i due poli della santificazione, per non perdere da una parte, cadendo nell'attivismo, le motivazioni interiori dell'apostolato e dall'altra, cadendo nell'intimismo, la pratica concreta della carità. Il contatto quotidiano con le persone e le loro situazioni, con le gioie e i dolori della gente, entra così nella preghiera del presbitero, che diviene atto di carità pastorale; a sua volta, la preghiera presbiterale - intessuta di parola di Dio e volti di persone - diviene alimento e sostegno del ministero, favorendo una dedicazione veramente «pastorale», che sa gettare sui fratelli uno sguardo di misericordia e di accoglienza. La spiritualità del presbitero diocesano, nell'assumere come elemento specifico la dedicazione in e ad una Chiesa particolare in nome e con la forza di Cristo pastore, capo e sposo, incarna in modo armonioso l'inscindibilità dell'azione e della preghiera, del dono di sé nelle molteplici relazioni ecclesiali e dell'accoglienza della grazia che viene da Cristo.

\section{InSERIMENTO NELLA ChieSA ATtRAVERSo OBBEDIENZA, POVERTÀ, CELIBATO}

La carità pastorale, infine, costituisce per il presbitero la maniera peculiare di vivere l'obbedienza, la povertà e il celibato. La trasposizione della spiritualità dei «tre voti» dalla tradizione monastica e religiosa al presbiterato, nella Chiesa di rito latino, non è un'operazione sorpassata né esteriore: mantiene invece tutta la sua validità e profondità, messe in risalto dall'ottica della carità pastorale. Globalmente considerati, obbedienza, povertà e celibato costituiscono una triplice conformazione a Cristo, obbediente al Padre fino alla morte di croce (Fil 2,8; Eb 5,8-9), povero per arricchirci (2 Cor 8,9) e misteriosamente identificato con i poveri (Mt 25,31-46), casto e celibe, avendo sposato la Chiesa (Mc 2,19-20 par.; Ef 5,21-33). Nel loro significato 
fondamentale, perciò, sono tre atteggiamenti che chiedono di essere riprodotti in ogni battezzato, se è vero che il primo sacramento realizza la condivisione del mistero di Cristo (Rom 6,3-6). Tutti i discepoli di Cristo sono quindi chiamati alla radicalità evangelica, ossia a vivere le loro relazioni nello stile dell'obbedienza, povertà e castità di Gesù: in un atteggiamento di libertà interiore, distacco dalle cose e dominio ordinato degli affetti e della sessualità; il grande criterio è, ancora una volta, la carità, il dono di sé.

Gesù, in definitiva, ha mostrato la possibilità per gli uomini di vivere nella storia la novità della radicale appartenenza a Dio nella totale dedicazione al suo Regno; le vocazioni che comportano scelte di radicale povertà, castità e obbedienza, assumono il servizio della testimonianza a tutti gli uomini della trascendenza del Regno: se è vero infatti che tutti i valori autenticamente umani sono altrettante pietre nella costruzione del Regno di Dio già a partire dalla storia, è altrettanto vero che nella sua pienezza il Regno trascende ogni realizzazione umana, anche la più elevata. Le vocazioni monastiche, religiose e laicali che, attraverso la consacrazione, accolgono il triplice dono della povertà, castità e obbedienza vissute in maniera radicale, offrono quindi una testimonianza di cui tutti hanno bisogno: Dio è l'unica grande ricchezza, è l'unica vera e duratura gioia, è il solo al quale conviene consegnare la propria libertà; quando Dio è tutto, allora le relazioni con gli altri, con la propria libertà e con le cose trovano significato, direzione e collocazione adeguata. Questa triplice testimonianza è un aiuto, per coloro che vivono altre vocazioni, a riferire tutte le relazioni a Dio, a tenerlo al centro della propria vita.

Tra la spiritualità dei «voti» dei consacrati e quella dei ministri ordinati non v'è coincidenza, ma vi sono motivi di convenienza. I presbiteri sono chiamati ad una piena conformazione a Cristo, non solo in quanto sono posti nella Chiesa, ma anche in quanto si collocano di fronte ad essa, agendo in persona di Cristo capo, sposo e pastore; come tali, essi si impegnano a vivere la povertà, la castità e l'obbedienza nella maniera loro propria, quella della carità pastorale. Nel dedicarsi stabilmente alla Chiesa, essi abbracciano Cristo obbediente al Padre, promettendo al vescovo «filiale rispetto e obbedienza», entrando con rispetto e spirito costruttivo nelle relazioni all'interno del presbiterio e del popolo di Dio; essi abbracciano Cristo sposo della Chiesa, vergine e casto, impegnandosi a vivere in un celibato che è occasione per un dono di sé ampio, ricco, generoso, «con cuore indiviso», aperto a tutti ; essi abbracciano Cristo povero che arricchisce molti, vivendo uno stile di distacco dalle cose e utilizzando ciò di cui dispongono per il loro ministero e la loro missione e facendosi in tal modo più attenti ai poveri . 
I presbiteri terranno presente che l'imitazione e la sequela di Cristo povero, casto e obbediente non è facile e va contro la mentalità mondana, dominata dagli «idoli»: la croce, quindi, fa parte essenziale della sequela e non è da interpretare - fino a prova contraria - come un incidente di percorso o l'indizio di una errata valutazione vocazionale. I dolorosi abusi in tutti questi campi richiedono tuttavia un profondo ripensamento - in parte già in atto - della formazione presbiterale seminaristica e permanente. Fa parte infine della spiritualità presbiterale l'integrazione degli insuccessi, dei fallimenti e delle delusioni nel proprio cammino, quali spine nella carne inseparabili dall'apostolato che il Signore lascia sussistere perché la sua grazia si manifesti pienamente nella debolezza dei ministri (2Cor 12,7-10). Sono le «persecuzioni» che Gesù stesso ha realisticamente connesso alla sequela, inquadrandole però in una gioia che non ha pari: il centuplo quaggiù e la vita eterna (Mc 10,30).

\section{BIBLIOGRAFIA}

Castellucci, E., Il ministero ordinato, Brescia 2006, 248-270.

Congar, Y., «L'idée du sacerdoce dans les textes de Vatican II», Verbum Caro 85 (1968) 11-15.

Denis, H., «La teologia del presbiterato da Trento al Vaticano II», in Y. Congar - J. Frisque (eds.), I preti. Formazione, ministero e vita, Roma 1970, 105-154.

Dianich, S., Teologia del ministero ordinato, Paoline, Roma 1984.

Dumont, C., «La 'charité pastorale' et la vocation au presbytérat. A propos de 1'Exhortation post-synodale Pastores dabo vobis», Nouvelle Revue Théologique 115 (1993) 211-226.

Frisque, J., «Théologie du sacerdoce selon Vatican II», Revue Nouvelle (1966) 481-496.

Grelot,P., «Il sacerdozio comune dei fedeli nel Nuovo Testamento», in Regole e tradizioni del cristianesimo primitivo, Casale Monferrato 1998, 115-131.

Penna, R., «Los ministerios en las primeras comunidades cristianas según el Nuevo Testamento», Seminarios 66 (2021) 11-33. https://doi.org/10.52039/ seminarios.v66i228.375. 